\title{
Consistent Abstractions of Affine Control Systems
}

\author{
George J. Pappas, Member, IEEE, and Slobodan Simić
}

\begin{abstract}
In this paper, we consider the problem of constructing abstractions of affine control systems that preserve reachability properties, and, in particular, local accessibility. In this framework, showing local accessibility of the higher level, abstracted model is equivalent to showing local accessibility of the, more detailed, lower level model. Given an affine control system and a smooth surjective map, we present a canonical construction for extracting an affine control system describing the trajectories of the abstracted variables. We then obtain conditions on the abstraction maps that render the original and abstracted system equivalent from a local accessibility point of view. Such consistent hierarchies of accessibility preserving abstractions of nonlinear control systems are then considered for various classes of affine control systems including linear, bilinear, drift free, and strict feedback systems.
\end{abstract}

Index Terms-Abstraction, affine control systems, hierarchies, local accessibility.

\section{INTRODUCTION}

A NATURAL approach for reducing the complexity of large scale systems places a hierarchical structure on the system architecture. For example, in the common two-layer planning and control hierarchies, the planning level uses a coarser system model than the lower control level. One of the main challenges in hierarchical systems is the extraction of a hierarchy of models at various levels of abstraction while preserving properties of interest.

Abstraction is also important in the analysis of complex systems. In the area of formal verification of concurrent systems, problems of exponential complexity are frequently encountered, and hierarchical system abstractions are used for complexity reduction [9], [16], [17]. For example, in order to verify that a given large scale system satisfies certain properties, one tries to extract a simpler but qualitatively equivalent abstracted system. Checking the desired property on the abstracted system should be equivalent or sufficient to checking the property on the original system. Depending on the property, special quotient systems which preserve the property of interest are constructed.

As a result, the notion of abstraction refers to grouping the system states into equivalence classes. A hierarchy can be thought of as a finite sequence of abstractions. Consistent abstractions are property preserving abstractions. Depending

Manuscript received March 28, 2001; revised November 4, 2001. Recommended by Associate Editor A. Bemporad. The work of G. J. Pappas was supported in part by DARPA ITO MoBIES F33615-00-C-1707, and by the National Science Foundation under Grant ITR CCR01-21431. The work of S. Simić was supported in part by NASA under Grant NAG-2-1039 and EPRI grant EPRI35352-6089.

G. J. Pappas is with the Department of Electrical Engineering, University of Pennsylvania, Philadelphia, PA 19104 USA (e-mail: pappasg@ee.upenn.edu).

S. Simić is with the Department of Electrical Engineering and Computer Science, University of California, Berkeley, CA 94720 USA (e-mail simic@eecs.berkeley.edu).

Publisher Item Identifier S 0018-9286(02)04767-0. on the cardinality of the resulting quotient space we may have discrete or continuous abstractions. With this notion of abstraction, the abstracted system is defined as the quotient system dynamics. In this spirit, abstractions of purely discrete-event systems have been formally considered in the computer science community [9], [16] based on the fundamental work of [17]. Similar work for discrete event systems has been also considered in the control community [7], [29], [30]. A related research area considers equivalent discrete abstractions of continuous or hybrid systems [2], [8], [14] as well as sufficient discrete abstractions of hybrid systems [4], [10], [23].

In previous work, we have focused on extracting continuous abstractions from continuous systems. In particular, in [21], a hierarchical framework for continuous control systems was conceptualized and formally proposed. In [20], easily checkable characterizations were obtained for constructing controllability preserving abstractions of linear control systems. This immediately resulted in a hierarchical controllability algorithm from which we recovered the best known controllability algorithm from numerical linear algebra [11], [15]. In the same spirit, in [19] we characterized stabilizability preserving abstractions of linear systems. The resulting hierarchical stabilizability algorithm recovers the stabilizability algorithm in [24].

In this paper, we extend our hierarchical approach to a significant class of nonlinear control systems that consists of affine control systems on smooth manifolds. ${ }^{1}$ In particular, we address the following problem.

Problem 1.1: Given an affine control system

$$
\dot{x}=F(x)+G(x) u \quad x \in \mathbb{R}^{n} \quad u \in \mathbb{R}^{m}
$$

and a smooth, surjective map $y=\Phi(x)$, where $\Phi: \mathbb{R}^{n} \rightarrow \mathbb{R}^{p}$, $p \leq n$, construct a control system

$$
\dot{y}=f(y)+g(y) v \quad y \in \mathbb{R}^{p} \quad v \in \mathbb{R}^{k}
$$

which can produce as trajectories all functions of the form $y(t)=\Phi(x(t))$, where $x(t)$ is a trajectory of (1). Furthermore, characterize smooth maps $\Phi$ for which (1) is locally accessible (controllable) if and only if (2) is locally accessible (controllable).

The surjective map $\Phi$ partitions the state space into equivalence classes. System (2) will be referred to as the abstraction of the more detailed model (1). It should be noted that the notion of abstraction in this paper is quite different from previous notions of state aggregation [5], [13], [26], and the more established notion of approximate model reduction [3], [28]. In model reduction, the input and output of the system are fixed, while the state

\footnotetext{
${ }^{1}$ A preliminary version of this work for analytic, drift-free systems appeared
} in [22]. 
dimension is reduced. The abstraction problem that we formulate does not require the input of the two systems to be the same. This is typical in planning and control hierarchies where, for example, the input at the kinematic level may be a velocity input, whereas the input at the dynamic level may be a torque input.

In [20], we extended the geometric notion of $\Phi$-related vector fields to control systems, which allowed us to push forward control systems through maps and obtain well defined control systems describing the abstracted dynamics. The fact that the abstraction map $\Phi$ sends trajectories of (1) to trajectories of (2) enabled us to propagate reachable sets from system (1) to system (2). Furthermore, in [20], we were able to provide constructive formulas for constructing linear abstractions of linear control systems.

In this paper, we provide a constructive method for extracting abstractions for affine control systems on smooth manifolds. Our method is the natural nonlinear generalization of the linear method provided in [20]. Furthermore, the method is natural in the sense that it constructs the smallest $\Phi$-related or abstracted control system. In addition, our method is structure preserving in the sense that the affine structure of our control systems is preserved throughout the abstraction process. Therefore, by repeating our construction, we can obtain a hierarchy, that is a finite sequence, of affine abstractions.

We then consider the problem of constructing abstractions while preserving the property of local accessibility [18]. We determine conditions on the map $\Phi$ under which local accessibility of the abstracted system (2) is equivalent to local accessibility of (1). Such conditions greatly reduce the complexity of determining local accessibility properties of nonlinear control systems, since rather than checking controllability of a large scale nonlinear system, we can construct a hierarchy of consistent abstractions and then check the local accessibility of systems which are much smaller in size. A property preserving hierarchy will then propagate the desired property along the sequence of abstractions from the simplest abstracted model to the original complex system.

The structure of this paper is as follows. In Section II, we review the results in [20] in the setting of linear systems. In Section III, we review some differential geometric concepts that are used in the paper, whereas in Section IV, we review some results from [20] that are used in this paper. In Section V, we provide methods for constructing abstractions of affine control systems. In Section VI, we characterize abstractions that preserve the property of local accessibility. This leads to hierarchical accessibility criteria which are considered for various classes of affine systems in Section VII. Finally, Section VIII discusses interesting directions for further research.

\section{LINEAR ABSTRACTIONS}

The main goal of this paper is to obtain nonlinear analogues of the results in [20]. We start our review of the results in [20] with a formal definition of linear abstractions.

Definition 2.1 [Linear Abstractions ([20])]: Consider the linear control systems

$$
\begin{array}{llll}
\left(\Sigma_{1}\right) & \dot{x}=A x+B u & x \in \mathbb{R}^{n} & u \in \mathbb{R}^{m} \\
\left(\Sigma_{2}\right) & \dot{y}=F y+G v & y \in \mathbb{R}^{p} & v \in \mathbb{R}^{k}
\end{array}
$$

and a surjective map $y=C x$. Then control system $\Sigma_{2}$ is called a $C$-abstraction or abstraction of system $\Sigma_{1}$ if system $\Sigma_{2}$ can produce as trajectories all functions of the form $y(t)=C x(t)$, where $x(t)$ is a trajectory of system $\Sigma_{1}$.

The above definition of abstraction relates the trajectories of the two systems. Note that system $\Sigma_{2}$ must capture all (output) trajectories of system $\Sigma_{1}$, but may also generate more trajectories. At the level of vector fields, we have the following notion.

Definition 2.2 (C-Related Linear Systems): Consider the linear time-invariant control systems

$$
\begin{array}{llll}
\left(\Sigma_{1}\right) & \dot{x}=A x+B u & x \in \mathbb{R}^{n} & u \in \mathbb{R}^{m} \\
\left(\Sigma_{2}\right) & \dot{y}=F y+G v & y \in \mathbb{R}^{p} & v \in \mathbb{R}^{k}
\end{array}
$$

and the linear, surjective map $y=C x$. Then, $\Sigma_{2}$ is $C$-related to $\Sigma_{1}$ if for all $x \in \mathbb{R}^{n}, u \in \mathbb{R}^{k}$, there exists $v \in \mathbb{R}^{l}$ such that

$$
C(A x+B u)=F C x+G v .
$$

The notion of $C$-related control systems simply states that system $\Sigma_{2}$ must be able to generate (using its control input $v \in \mathbb{R}^{l}$ ), the image under $C$ of any tangent vector that system $\Sigma_{1}$ may generate at any point $x \in \mathbb{R}^{n}$, and given any control input $u \in \mathbb{R}^{k}$. The connection between $C$-abstractions and $C$-related systems is given by the following theorem.

Theorem 2.3 (C-Abstractions and C-Related Systems [20])

: Consider the linear time-invariant control systems

$$
\begin{array}{llll}
\left(\Sigma_{1}\right) & \dot{x}=A x+B u & x \in \mathbb{R}^{n} & u \in \mathbb{R}^{m} \\
\left(\Sigma_{2}\right) & \dot{y}=F y+G v & y \in \mathbb{R}^{p} & v \in \mathbb{R}^{k}
\end{array}
$$

and the linear, surjective map $y=C x$. Then, $\Sigma_{2}$ is a $C$-abstraction of $\Sigma_{1}$ if and only if $\Sigma_{2}$ is $C$-related to $\Sigma_{1}$.

Given $C$-abstractions and $C$-related systems, it is clearly advantageous to work with $C$-related systems since they potentially offer algebraic methods for constructing abstractions. In particular, the following proposition gives us a canonical construction in order to generate $C$-related linear abstractions.

Theorem 2.4 [Canonical Construction ([20])] : Consider the linear system

$$
\left(\Sigma_{1}\right) \quad \dot{x}=A x+B u
$$

and a surjective map $y=C x$. Let

$$
\left(\Sigma_{2}\right) \quad \dot{y}=F y+G v
$$

be the system where

$$
\begin{aligned}
& F=C A C^{+} \\
& G=\left[\begin{array}{lllll}
C B & C A v_{1} & \ldots & C A v_{r}
\end{array}\right]
\end{aligned}
$$

where $C^{+}$is the Moore-Penrose pseudoinverse of $C$, and $v_{1}, \ldots, v_{r}$ span $\operatorname{Ker}(C)$. Then $\Sigma_{2}$ is $C$-related to $\Sigma_{1}$.

Note that by Proposition 2.5, given any linear control system, and any full-row rank matrix $C$, there always exists another linear control system which is $C$-related to it. In addition to propagating trajectories from the original to the abstracted system, we are also interested in propagation of other properties such as controllability. From linear systems theory we know 
that the reachable space from the origin for system $\left(\Sigma_{1}\right)$ is given by $\mathcal{R}(A, B)=\operatorname{Im}\left[\begin{array}{llll}B & A B & \ldots & A^{n-1} B\end{array}\right]$. In particular, system $\Sigma_{1}$ is controllable if and only if $\mathcal{R}(A, B)=\mathbb{R}^{n}$. As an immediate corollary of Theorem 2.3 we obtain that $C \mathcal{R}(A, B) \subseteq \mathcal{R}(F, G)$, and, in particular, if $\Sigma_{1}$ is controllable then $\Sigma_{2}$ is controllable.

In order to propagate controllability from the abstracted linear system $\Sigma_{2}$ to the original system $\Sigma_{1}$, conditions must be placed on the abstracting map $y=C x$, resulting in consistent abstractions [20]. With respect to controllability, the following theorem characterizes consistent linear abstractions.

Theorem 2.5 [Controllability Preserving Abstractions ([20])]: Consider the linear system

$$
\left(\Sigma_{1}\right) \quad \dot{x}=A x+B u
$$

and surjective map $y=C x$. Let

$$
\left(\Sigma_{2}\right) \quad \dot{y}=F y+G v
$$

be the $C$-related system where

$$
\begin{aligned}
& F=C A C^{+} \\
& G=\left[\begin{array}{llll}
C B & C A v_{1} & \ldots & C A v_{r}
\end{array}\right]
\end{aligned}
$$

where $C^{+}$is the Moore-Penrose pseudoinverse of $C$ and $v_{1}, \ldots, v_{r}$ span $\operatorname{Ker}(C)$. Furthermore, if

$$
\operatorname{Ker}(C) \subseteq \mathcal{R}(A, B)
$$

then $\Sigma_{1}$ is controllable if and only if $\Sigma_{2}$ is controllable.

The condition $\operatorname{Ker}(C) \subseteq \mathcal{R}(A, B)$ suggests that in order to abstract away some dynamics (captured by $\operatorname{Ker}(C)$ ) while preserving controllability, one would have to ensure the ignored dynamics are controllable. From the assumptions of Theorem 2.5 , it is easy to see that a controllability preserving linear abstraction always exists if $B \neq 0$, since we can always choose matrix $C$ satisfying $\operatorname{Ker}(C)=\operatorname{Im}[B]$. Therefore the controllability preserving condition serves as a guideline for choosing our abstracting matrix $C$.

The goal of this paper is to develop similar results for nonlinear, affine control systems of the form $\dot{x}=f(x)+g(x) u$. In particular, we are interested in generalizing the canonical construction of Theorem 2.4 for affine control systems. Furthermore, given that most results for nonlinear systems are local in nature, rather than propagating global controllability, we focus on the property of local accessibility, and obtain the nonlinear analogue of Theorem 2.5. In order to achieve this, we must rely on the differential geometric methods for accessibility of nonlinear systems.

\section{GEOMETRIC PRELIMINARIES}

We begin by recalling some definitions from differential geometry ([1], [18]). Let $M$ be a differentiable manifold, and denote by $T_{p} M$ the tangent space of $M$ at $p \in M$. Let $T M=$ $\bigcup_{p \in M} T_{p} M$ be the tangent bundle of $M$, and let $\pi$ be the canonical projection map $\pi: T M \rightarrow M$. Recall, for instance, that $T_{p} \mathbb{R}^{n}=\mathbb{R}^{n}$, and that $T \mathbb{R}^{n}=\mathbb{R}^{n} \times \mathbb{R}^{n}$. Throughout the paper, the reader can keep $\mathbb{R}^{n}$ as a model manifold without loss of any of the main ideas. Given a smooth map $\Phi: M \rightarrow N$ between smooth manifolds $M$ and $N$, the tangent map $T_{p} \Phi: T_{p} M \longrightarrow$ $T_{\Phi(p)} N$ pushes forward tangent vectors from $T_{p} M$ to $T_{\Phi(p)} N$. The union of all tangent maps $T_{p} \Phi$ is denoted by $T \Phi$. Recall that if both $M$ and $N$ are euclidean spaces, then $T \Phi$ is just the total derivative of $\Phi$. In this paper, we will be concerned with maps $\Phi: M \rightarrow N$ which are surjective submersions. In such cases, we will think of $N$ as an embedded submanifold of $M$. As a model example to keep in mind, take $M=\mathbb{R}^{n}$, $N=\mathbb{R}^{k}=\mathbb{R}^{k} \times\{\mathbf{0}\} \subset \mathbb{R}^{n}$, where $k<n$, and $\Phi$ is the projection to the first $k$ coordinates.

A vector field on a manifold $M$ is a smooth map $X: M \rightarrow T M$ which assigns to each point $p \in M$ a tangent vector $X(p) \in T_{p} M$. An integral curve of a vector field $X$ is a smooth curve $c: I \subseteq \mathbb{R} \rightarrow M$ that satisfies $T_{t} c=X(c(t))$ for all $t \in I$. Given two vector fields $X$ and $Y$ on $M$, by $[X, Y]$ we denote their usual Lie bracket.

A distribution $\Delta$ on $M$ assigns to each $p \in M$ a subspace of $T_{p} M$. A distribution generated by vector fields $X_{1}, \ldots, X_{k}$ is given by $\Delta=\operatorname{span}\left\{X_{1}, X_{2}, \ldots, X_{k}\right\}$. The dimension of $\Delta$ at $p \in M$, denoted by $\operatorname{dim}(\Delta(p))$, is then $\operatorname{dim}\left(\operatorname{span}\left\{X_{1}(p), X_{2}(p), \ldots, X_{k}(p)\right\}\right)$. Regular distributions require the dimension of the distribution to be independent of $p \in M$. A vector field $X$ belongs to a distribution $\Delta$ if $X(p) \in \Delta(p)$ at each $p \in M$.

Given two smooth distributions $\Delta_{1}$ and $\Delta_{2}$, we define the distribution $\left[\Delta_{1}, \Delta_{2}\right]$ by declaring $\left[\Delta_{1}, \Delta_{2}\right](p)$ to be the subspace of $T_{p} M$ generated by vectors of the form $\left[X_{1}, X_{2}\right](p)$ where $X_{1}, X_{2}$ are smooth vector fields belonging in $\Delta_{1}$ and $\Delta_{2}$ respectively. Given a distribution $\Delta, \operatorname{Lie}(\Delta)$ is the Lie algebra generated by $\Delta$. It is obtained by taking the span of iterated Lie brackets of vector fields in $\Delta$.

Given a vector field $X$ on manifold $M$ and a smooth map $\Phi: M \rightarrow N$, not necessarily a diffeomorphism, the push forward of $X$ by $T \Phi$ is generally not a well-defined vector field on $N$. This leads to the concept of $\Phi$-related vector fields.

Definition 3.1 ( $\Phi$-Related Vector Fields [1], [18]): Let $X$ and $Y$ be vector fields on manifolds $M$ and $N$, respectively, and $\Phi: M \rightarrow N$ be a smooth map. Then, $Y$ is $\Phi$-related to $X$ if for every $p \in M$

$$
T \Phi(X(p))=Y(\Phi(p))
$$

If $\Phi$ is a smooth surjection from $M$ to $N$, then given a vector field $X$ on a manifold $M$, the push forward of $X$ by $T \Phi$ is a well defined vector field on $N$ only if $T_{p_{1}} \Phi\left(X\left(p_{1}\right)\right)=T_{p_{2}} \Phi\left(X\left(p_{2}\right)\right)$ whenever $\Phi\left(p_{1}\right)=\Phi\left(p_{2}\right)$ for any two points $p_{1}, p_{2} \in M$. The following well-known theorem gives us a condition on the integral curves of two $\Phi$-related vector fields.

Theorem 3.2 ( $\Phi$-Related Vector Fields [1], [18]): Let $X$ and $Y$ be vector fields on $M$ and $N$ respectively and let $\Phi: M \rightarrow N$ be a smooth map. Then, vector fields $X$ and $Y$ are $\Phi$-related if and only if for every integral curve $c$ of $X, \Phi(c)$ is an integral curve of $Y$.

Even though $\Phi$-relatedness of vector fields is a rather restrictive condition, this is not the case for control systems. In order to have global definitions of control systems ([6], [18]), we shall need the concept of fiber bundles. 
Definition 3.3 (Fiber Bundles): A fiber bundle is a quintuple ( $B, M, \pi, U,\left\{O_{i}\right\}_{i \in I}$ ) where $B, M, U$ are smooth manifolds called the total space, the base space and the standard fiber, respectively. The map $\pi: B \rightarrow M$ is a surjective submersion and $\left\{O_{i}\right\}_{i \in I}$ is an open cover of $M$ such that for every $i \in I$ there exists a diffeomorphism $\Psi_{i}: \pi^{-1}\left(O_{i}\right) \rightarrow O_{i} \times U$ satisfying $\pi_{o} \circ \Psi_{i}=\pi$ where $\pi_{o}$ is the projection from $O_{i} \times U$ to $O_{i}$ (local triviality condition). The submanifold $\pi^{-1}(p)$ is called the fiber at $p \in M$.

If all the fibers are vector spaces of constant dimension, then the fiber bundle is called a vector bundle. If all the fibers are affine spaces then the fiber bundle is called an affine bundle.

The tangent bundle of a smooth manifold is an example of a fiber (vector) bundle. Some others are as follows.

Example 3.4 (Trivial Fiber Bundle): If $B=M \times U$ and $\pi: B \rightarrow M$ is the projection to the second coordinate, $(x, u) \longmapsto$ $x$, then the five-tuple $(B, M, \pi, U,\{M\})$ is called the trivial fiber bundle over $M$ with fiber $U$. For example, the 2-torus is a trivial fiber bundle over the circle $S^{1}$ with fiber $S^{1}$. Locally, every fiber bundle looks like the trivial one [1].

Example 3.5 (Distributions): Every distribution $\Delta$ can be regarded as a vector bundle by taking $B$ to be the union of all $\Delta(p)$ and defining the projection by $\pi(v)=p$ whenever $v \in \Delta(p)$. The fiber is $\mathbb{R}^{k}$, where $k=\operatorname{dim} \Delta$. The local triviality condition means that $\Delta$ is locally spanned by $k$ linearly independent vector fields.

If $\mathcal{A}$ is an affine bundle on $M$, then locally there exist a vector field $X_{0}$ and a distribution $\Delta$ such that $\mathcal{A}=X_{0}+\Delta$. If $\Delta$ is generated by vector fields $X_{1}, \ldots, X_{k}$ then $\mathcal{A}=X_{0}+$ $\operatorname{span}\left\{X_{1}, X_{2}, \ldots, X_{k}\right\}$. Formally, $B$ is the union of all affine spaces $X_{0}(p)+\Delta(p), \pi(v)=p$ for all $v \in \mathcal{A}(p)$, the fiber $U$ is an arbitrary but fixed affine $k$-dimensional subspace of $\mathbb{R}^{n}$ where $n=\operatorname{dim} M$.

Example 3.6: Consider the following (affine) control system on $M=\mathbb{R}^{2}$ :

$$
\begin{aligned}
& \dot{x}=1-u y \\
& \dot{y}=u x .
\end{aligned}
$$

Then at each point $\left(x_{0}, y_{0}\right)$, the set of all possible tangent directions is a straight line in $\mathbb{R}^{2}$ (considered as the tangent space to $M$ at $\left.\left(x_{0}, y_{0}\right)\right)$ given by the equation $x_{0}(x-1)+y_{0} y=0$. Note that this line does not pass through the origin which is why it forms an affine subspace. Here, $X_{0}=(1,0)^{T}$ and $X_{1}(x, y)=$ $-(y, x)^{T}$.

We will denote the Lie algebra generated by $\mathcal{A}$ by $\operatorname{Lie}(\mathcal{A})$. It is obtained by taking the span of all iterated Lie brackets of vector fields in $\mathcal{A}$. For simplicity, we will abuse the notation and use $\operatorname{Lie}(\mathcal{A})$ also to denote the distribution given by $\{X(p): X \in$ $\operatorname{Lie}(\mathcal{A})\}$.

\section{CONTROL SySTEM ABSTRACTIONS}

Definition 3.1 and Theorem 3.2 capture the essence of Problem 1.1, but for vector fields. The restrictive nature of Theorem 3.2 is due to the deterministic nature of vector fields. The nondeterministic nature of control systems, however, allows us to remove such restrictions. In [20], Definition 3.1 and Theorem 3.2 were extended to control systems. We now briefly review some of the results of those papers. We first begin with a global definition of control systems.

Definition 4.1 (Control Systems [6], [18]) : A control system $S=(U, F)$ consists of a fiber bundle $\pi: U \rightarrow M$ and a smooth map $F: U \rightarrow T M$ which is fiber preserving, that is $\pi^{\prime} \circ F=\pi$ where $\pi^{\prime}: T M \rightarrow M$ is the tangent bundle projection. Given a control system $S=(U, F)$, the control bundle $\mathcal{B}$ of $S$ is naturally defined pointwise by $\mathcal{B}(p)=F\left(\pi^{-1}(p)\right)$ for all $p \in M$. A control system is called affine if the control bundle $\mathcal{B}$ is an affine bundle.

The base manifold $M$ of the control bundle is the state space and the fibers $\pi^{-1}(p)$ can be thought of as the state dependent control spaces. Given the state $p$ and the input, the map $F$ selects a tangent vector from $\mathcal{B}(p) \subseteq T_{p} M$. The notion of trajectories of control systems in this context is now given.

Definition 4.2 (Trajectories of Control Systems): A smooth curve $c: I \rightarrow M$ is called a trajectory of the control system $S=(U, F)$ if there exists a curve $c^{U}: I \rightarrow U$ satisfying

$$
\begin{aligned}
\pi\left(c^{U}\right) & =c \\
T_{t} c & =F\left(c^{U}\right) .
\end{aligned}
$$

In local coordinates, Definition 4.2 simply says that a trajectory of a control system is a curve $x(t)$ for which there exists a function $u(t)$ satisfying, $\dot{x}(t)=F(x(t), u(t))$. Note that even though Definition 4.2 assumes $c$ to be smooth, the bundle curve $c^{U}$ is not necessarily smooth. The definition, therefore, allows nonsmooth control inputs as long as the projection $\pi\left(c^{U}\right)=c$ is smooth.

We now consider abstractions of control systems. An abstraction is a map $\Phi: M \rightarrow N$ which we will assume to be a surjective, smooth submersion. ${ }^{2}$ We can now define $\Phi$-related control systems in a manner similar to Definition 3.1 for vector fields.

Definition 4.3 ( $\Phi$-Related Control Systems) : Let $S_{M}=\left(U_{M}, F_{M}\right)$ with $\pi_{M}: U_{M} \rightarrow M$ and $S_{N}=\left(U_{N}, F_{N}\right)$ with $\pi_{N}: U_{N} \rightarrow N$ be two control systems. Let $\Phi: M \rightarrow N$ be a smooth map. Let $\mathcal{B}_{M}$ and $\mathcal{B}_{N}$ be the control bundles associated with control systems $S_{M}$ and $S_{N}$ respectively. Then $S_{N}$ is $\Phi$-related to $S_{M}$ if for every $p \in M$

$$
T_{p} \Phi\left(\mathcal{B}_{M}(p)\right) \subseteq \mathcal{B}_{N}(\Phi(p))
$$

Control system $S_{N}$ will be referred to as an abstraction of control system $S_{M}$ ([20]). Note that many control systems $S_{N}$ may be $\Phi$-related to $S_{M}$ as the set of tangent vectors on $N$ that must be captured, can be generated using many control parameterizations.

It is straightforward to show that $\Phi$-relatedness of control systems indeed generalizes Definition 3.1 [20]. Furthermore, if $\mathcal{B}_{N_{1}}$ and $\mathcal{B}_{N_{2}}$ satisfy condition (4), then $\mathcal{B}_{N_{1}} \cap \mathcal{B}_{N_{2}}$ also satisfies condition (4). This suggests that there exists a minimal system $S_{M}$, up to control parameterization, that is $\Phi$-related to $S_{M}$. The

\footnotetext{
${ }^{2}$ Note that any map $\Phi$ gives rise to an equivalence relation by defining states $x$ and $y$ equivalent if $\Phi(x)=\Phi(y)$. In order for the resulting quotient space to have a manifold structure, the equivalence relation must be regular [1].
} 
minimal system naturally corresponds to the case where condition (4) becomes an equality, or equivalently when the following diagram commutes:

$\begin{array}{ccc}M & \stackrel{\Phi}{\rightarrow} & N \\ \mathcal{B}_{M} \downarrow & & \downarrow \mathcal{B}_{N} \\ \mathcal{F}(M) & \stackrel{T \Phi}{\longrightarrow} & \mathcal{F}(N),\end{array}$

where $\mathcal{F}(M)$ is the space of fiber subbundles of $T M$.

In contrast to the restrictive conditions of Theorem 3.2, the following straightforward proposition, shows that every control or dynamical system is $\Phi$-related to some control system for any map $\Phi$.

Proposition 4.4 ([20]): Given any control system $S_{M}=$ $\left(U_{M}, F_{M}\right)$ and any smooth map $\Phi: M \rightarrow N$, then there always exists a control system $S_{N}=\left(U_{N}, F_{N}\right)$ which is $\Phi$-related to $S_{M}$.

The following theorem generalizes Theorem 3.2 to control systems.

Theorem 4.5 ( $\Phi$-Related Control Systems [20]) : Let $S_{N}=$ $\left(U_{N}, F_{N}\right)$ and $S_{M}=\left(U_{M}, F_{M}\right)$ be two control systems and $\Phi: M \rightarrow N$ be a smooth map. Then $S_{N}$ is $\Phi$-related to $S_{M}$ if and only if for every trajectory $c_{M}$ of $S_{M}, \Phi\left(c_{M}\right)$ is a trajectory of $S_{N}$.

Because of Theorem 4.5, throughout this paper, we can equivalently say that $S_{N}$ is an abstraction of $S_{M}$ or that $S_{N}$ is $\Phi$-related to $S_{M}$. If $\Sigma_{S_{M}}$ and $\Sigma_{S_{N}}$ denote all trajectories of control systems $S_{M}$ and $S_{N}$, respectively, then Theorem 4.5 simply states that $S_{N}$ is $\Phi$-related to $S_{M}$ if and only if $\Phi\left(\Sigma_{S_{M}}\right) \subseteq$ $\Sigma_{S_{N}}$. The abstracted system therefore overapproximates the abstracted trajectories of the original system which may result in trajectories that the abstracted system $S_{N}$ may generate but are infeasible in the original model $S_{M}$.

Even though Definition 4.3 and Theorem 4.5 for control systems remove the tight restrictions of Definition 3.1 and Theorem 3.2 for dynamical systems, the challenge now becomes providing methods for constructing abstractions of control systems. This is the objective of Section V.

\section{AbStRaCtion CONSTRUCTION}

The results we reviewed in Section IV were true for general control bundles, including affine bundles. In this section, we present a canonical way of constructing abstractions for affine control systems. Therefore, from this point on, we assume that all objects are smooth and all control bundles are affine.

Let $S_{M}=\left(U_{M}, F_{M}\right)$ be a control system on a manifold $M$. Denote the affine control bundle of $S_{M}$ by $\mathcal{A}_{M}$. This is an affine subbundle of $T M$, so there exists a vector field $X_{M}$ on $M$ and a distribution $\Delta_{M}$ on $M$ such that

$$
\mathcal{A}_{M}=X_{M}+\Delta_{M}
$$

We say that $\Delta_{M}$ is the distribution associated with $\mathcal{A}_{M}$. Let $\Phi: M \rightarrow N$ be a surjective submersion, where $N$ is an embedded submanifold of $M$. Denote by $K$ the vector subbundle of $T M$ defined as

$$
K=\operatorname{Ker}(T \Phi)=\{v \in T M: T \Phi(v)=0\} .
$$

Since $\Phi$ is a submersion, the distribution $K$ has constant dimension $k=m-n$ everywhere, where $m=\operatorname{dim} M$ and $n=\operatorname{dim} N$. Furthermore, $K$ is an integrable distribution. Denote the foliation that is tangent to $K$ by $\mathcal{K}$.

Our goal is to construct the smallest control system $S_{N}$ on $N$ which is $\Phi$-related to $S_{M}$. We will accomplish this by constructing the smallest $K$-invariant affine subbundle $\tilde{\mathcal{A}}_{M}$ of $T M$ containing $\mathcal{A}_{M}$ whose associated distribution contains $K$, and taking $S_{N}$ to be any control system whose control bundle equals $T \Phi\left(\tilde{\mathcal{A}}_{M}\right)$.

A fiber bundle $\mathcal{B}$ over $M$ is called $X$-invariant, for some smooth vector field $X$ with local flow $\phi_{t}$, if $T_{p} \phi_{t}(\mathcal{B}(p)) \subset$ $\mathcal{B}\left(\phi_{t}(p)\right)$, for all $p \in M$ and $t \in \mathbb{R}$ for which both sides are defined. For a distribution $K$, we say that $\mathcal{B}$ is $K$-invariant, if it is $X$-invariant for every vector field $X$ in $K$.

Proposition 5.1: Let $\mathcal{A}$ be an affine subbundle of $T M$, where $\mathcal{A}(p)=X_{0}(p)+\Delta(p)$, for some vector field $X_{0}$ on $M$ and distribution $\Delta$ on $M$. Let $X$ be a vector field on $M$. Then $\mathcal{A}$ is $X$-invariant if and only if

$$
[X, \mathcal{A}] \subset \Delta .
$$

Proof: $(\Rightarrow)$ : Assume $\mathcal{A}$ is $X$-invariant. Denote the local flow of $X$ by $\phi_{t}$ and let $Y$ be any vector field in $\Delta$. Then, for every $p \in M$ and $t$

$$
T \phi_{-t}\left\{X_{0}\left(\phi_{t}(p)\right)+Y\left(\phi_{t}(p)\right)\right\} \in \mathcal{A}(p) .
$$

Subtracting $X_{0}(p)+Y(p)$ from the left hand side, dividing by $t$, and letting $t \rightarrow 0$, we obtain $\left[X, X_{0}+Y\right](p) \in \Delta(p)$. Therefore, $[X, \mathcal{A}] \subset \Delta$.

$(\Leftarrow)$ : Since $[X, \Delta] \subset[X, \mathcal{A}] \subset \Delta$, by a standard result in differential geometry [18], it follows that the distribution $\Delta$ is $X$-invariant. Similarly, we obtain that the distribution $\mathbb{R} X_{0} \oplus \Delta$ is $X$-invariant. Therefore, for every $p \in M$ and $t \in \mathbb{R}$ for which $\phi_{t}(p)$ is defined

$$
T \phi_{-t}\left(X_{0}\left(\phi_{t}(p)\right)=\alpha(p, t) X_{0}(p) \bmod \Delta\right.
$$

for some real-valued function $\alpha$. That is, $T \phi_{-t}\left(X_{0}\left(\phi_{t}(p)\right)-\right.$ $\alpha(p, t) X_{0}(p) \in \Delta(p)$. Since $\left.(d / d t)\right|_{0} T \phi_{-t}\left(X_{0}\left(\phi_{t}(p)\right)=\right.$ $\left[X, X_{0}\right](p) \in \Delta(p)$, it is easy to see that $\dot{\alpha}(p, 0)=0$, for all $p$. However, $\alpha$ is a 1-cocyle over the flow of $X$, i.e., $\alpha(p, t+s)=$ $\alpha(p, t) \alpha\left(\phi_{t}(p), s\right)$, so $\dot{\alpha}(p, t)=\alpha(p, t) \dot{\alpha}\left(\phi_{t}(p), 0\right)=0$. Since $\alpha(p, 0)=1$, it follows that $\alpha(p, t)$ is identically equal to one. This implies that $T \phi_{t}\left(X_{0}\right) \in \mathcal{A}$, as desired.

Definition 5.2 (Canonical Construction in $M$ ) : Given $\mathcal{A}_{M}$ and $K$ as above, let $\tilde{\Delta}_{M}$ be the smallest $K$-invariant distribution containing $\Delta_{M}, K$, and $\left[K, X_{M}\right]$ (see Fig. 1). Therefore, $\tilde{\Delta}_{M}$ is generated by

$$
L_{M} \cup\left[K, L_{M}\right] \cup\left[K,\left[K, L_{M}\right]\right] \cup \cdots
$$

where $L_{M}=\Delta_{M} \cup K \cup\left[K, X_{M}\right]$. Define the $\tilde{\mathcal{A}}_{M}$ as

$$
\tilde{\mathcal{A}}_{M}=X_{M}+\tilde{\Delta}_{M} .
$$

The affine bundle $\tilde{\mathcal{A}}_{M}$ is called the canonical bundle associated with $\mathcal{A}_{M}$ and $K$. 


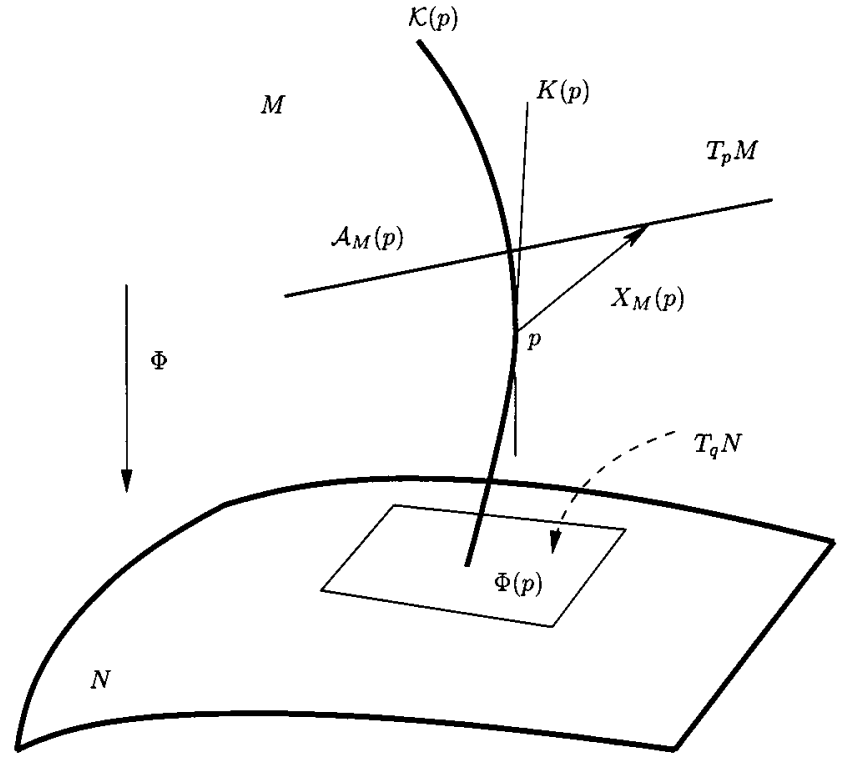

Fig. 1. Construction of $\tilde{\mathcal{A}}_{M}$.

The following proposition establishes the invariance properties needed for our construction.

Proposition 5.3 ( $K$-Invariance and $\Phi$-Relatedness):

a) The affine bundle $\tilde{\mathcal{A}}_{M}$ of Definition 5.2 contains $\mathcal{A}_{M}$, it is $K$-invariant, and its associated distribution $\tilde{\Delta}_{M}$ contains $K$. Moreover, it is the smallest affine bundle with these properties.

b) If $\Phi\left(p_{0}\right)=\Phi\left(p_{1}\right)$ for some $p_{0}, p_{1} \in M$, then

$$
\left(T_{p_{0}} \Phi\right)\left(\tilde{\mathcal{A}}_{M}\left(p_{0}\right)\right)=\left(T_{p_{1}} \Phi\right)\left(\tilde{\mathcal{A}}_{M}\left(p_{1}\right)\right) .
$$

\section{Proof:}

a) Clearly $\mathcal{A}_{M} \subset \tilde{\mathcal{A}}_{M}$. $K$-invariance follows from the construction of $\tilde{\Delta}_{M}$, the inclusion

$$
\left[K, \tilde{\mathcal{A}}_{M}\right]=\left[K, X_{M}\right]+\left[K, \tilde{\Delta}_{M}\right] \subset \tilde{\Delta}_{M}
$$

and Proposition 5.1.

To show that $\tilde{\mathcal{A}}_{M}$ is the smallest affine bundle with these properties, let $\mathcal{A}$ be another $K$-invariant affine bundle containing $\mathcal{A}_{M}$ whose associated distribution contains $K$. Then $\mathcal{A}=X_{M}+\Delta$, for some distribution $\Delta$ containing $\Delta_{M}$. By $K$-invariance, $[K, \Delta] \subset[K, \mathcal{A}] \subset \Delta$, so $\Delta$ is $K$-invariant. Similarly, $\left[K, X_{M}\right] \subset \Delta$. Since $\widetilde{\Delta}_{M}$ is by construction the smallest $K$-invariant distribution containing $\Delta_{M}, K$, and $\left[K, X_{M}\right]$, it follows that $\tilde{\Delta}_{M} \subset \Delta$, hence $\tilde{\mathcal{A}}_{M} \subset \mathcal{A}$.

b) By the Frobenius Theorem, locally each leaf of $\mathcal{K}$ is a plane $x_{k+1}=$ constant, $\ldots, x_{m}=$ constant, $N$ is the plane $\mathbb{R}^{n} \times \mathbf{0}$ in $\mathbb{R}^{m}$, and $\Phi$ is the projection $\left(x_{1}, \ldots, x_{m}\right) \mapsto\left(x_{1}, \ldots, x_{n}, 0, \ldots, 0\right)$. Assume $p_{0}$ and $p_{1}$ both lie in one such foliation chart [1] of $\mathcal{K}$. Since $\tilde{\mathcal{A}}_{M}$ is $K$-invariant and (in the same chart) $T \Phi=\operatorname{diag}\left(I_{n \times n}, \mathbf{0}\right)$, where $I_{n \times n}$ is the $n \times n$ identity matrix, it is easy to see that (b) holds. If $p_{1}$ is not in the same foliation chart as $p_{0}$, we can apply a similar argument to a finite sequence of foliation charts covering a path (in the leaf $\mathcal{K}\left(p_{0}\right)$ ) connecting $p_{0}$ and $p_{1}$.

The above proposition ensures well posedness of the following definition which summarizes our canonical construction for extracting affine abstractions from affine control systems.

Definition 5.4 (Canonical Construction on $N$ ) : Let $S_{M}=$ $\left(U_{M}, F_{M}\right)$ be a control system on a manifold $M$ with affine control bundle

$$
\mathcal{A}_{M}=X_{M}+\Delta_{M}
$$

Let $\Phi: M \rightarrow N$ be a surjective submersion, where $N$ is an embedded submanifold of $M$. Denote by $K$ the vector subbundle of $T M$ defined by (6). Define the affine distribution $\tilde{\mathcal{A}}_{M}$ by

$$
\tilde{\mathcal{A}}_{M}=X_{M}+\tilde{\Delta}_{M}
$$

where $\tilde{\Delta}_{M}$ is generated by (7). The affine bundle $\mathcal{A}_{N}$ on $N$ defined by

$$
\mathcal{A}_{N}(q)=T_{p} \Phi\left(\tilde{\mathcal{A}}_{M}(p)\right)
$$

for any $p \in \Phi^{-1}(q)$, is said to be canonically $\Phi$-related to $\mathcal{A}_{M}$. Any control system $S_{N}=\left(U_{N}, F_{N}\right)$ with control bundle $\mathcal{A}_{N}$ is said to be canonically $\Phi$-related to $S_{M}$.

Theorem 5.5 (Canonically $\Phi$-Related Systems) : The bundle $\mathcal{A}_{N}$ of Definition 5.4 is the smallest bundle on $N$ which is $\Phi$-related to $\mathcal{A}_{M}$.

Proof: That $\mathcal{A}_{N}$ is $\Phi$-related to $\mathcal{A}_{M}$ follows from its construction and Proposition 5.3. To show that it is the smallest, assume $\mathcal{B}$ is another bundle on $N \Phi$-related to $\mathcal{A}_{M}$. Let $\tilde{\mathcal{B}}=$ $(T \Phi)^{-1}(\mathcal{B})$. Then $\tilde{\mathcal{B}}$ clearly contains $\mathcal{A}_{M}$ and is $K$-invariant. Therefore, by Proposition 5.3, $\tilde{\mathcal{A}}_{M} \subset \tilde{\mathcal{B}}$. It is then immediate that $\mathcal{A}_{N}=T \Phi\left(\tilde{\mathcal{A}}_{M}\right) \subset T \Phi(\tilde{\mathcal{B}})=\mathcal{B}$, which proves the minimality of $\mathcal{A}_{N}$.

Definitions 5.2 and 5.4, and Theorem 5.5 provide us with a constructive method to construct $\Phi$-related systems. Furthermore, the construction is natural since it generates the smallest such system. We shall apply the canonical construction to various classes of affine systems in Section VII. In Section VI, we consider the relationship between $\Phi$-related control systems regarding accessibility and reachability properties.

\section{ACCESSIBILITY EQUIVALENCE}

In addition to constructing abstractions of nonlinear systems, we are also interested in preserving properties of interest between the original and abstracted model. In [20], we focused on controllability of linear control systems. In this paper, we focus on local accessibility for affine control systems.

We first recall some standard definitions for reachable sets. Consider a control system $S_{M}=\left(U_{M}, F_{M}\right)$, let $V \subset M$ be a neighborhood of $p \in M$, and consider time $T>0$. The reachable set from $p$ at time $T$, denoted $\mathcal{R}_{S_{M}}^{V}(p, T)$, is the set of points that can be reached from $p$ with trajectories of $S_{M}$ that remain within $V$ for all $0<t \leq T$. In our definition of control systems, the reachable set is formally expressed as follows.

Definition 6.1 (Reachable Sets [18]) : Let $S_{M}=\left(U_{M}, F_{M}\right)$ be a control system on a manifold $M$. Given a neighborhood $V$ 
of $p \in M$, define the reachable set in time $T>0$ as

$$
\begin{aligned}
\mathcal{R}_{S_{M}}^{V}(p, T)= & \left\{p^{\prime} \in M \mid \exists c^{U}:[0, T] \rightarrow U_{M}\right. \text { with } \\
& c=\pi\left(c^{U}\right) \quad c(0)=p \quad c(T)=p^{\prime} \text { and } \\
& \forall 0<t \leq T, \quad c(t) \in V\} .
\end{aligned}
$$

The reachable set from $p$ up to time $T$ is defined as

$$
\mathcal{R}_{T}^{V}\left(p, S_{M}\right)=\bigcup_{0<\tau \leq T} \mathcal{R}_{S_{M}}^{V}(p, \tau)
$$

Using the above definition of reachable sets, we can now define various notions of local accessibility.

Definition 6.2 (Local Accessibility [18]): A control system $S_{M}=\left(U_{M}, F_{M}\right)$ on a manifold $M$ is said to be the following.

a) Locally accessible at $p \in M$ if for every neighborhood $V$ of $p$ and every $T>0, \mathcal{R}_{T}^{V}\left(p, S_{M}\right)$ contains a nonempty, open set of $M$.

b) Locally accessible if it is locally accessible at every $p \in$ $M$.

c) Symmetrically locally accessible at $p \in M$ if it is locally accessible at $p \in M$, and $\mathcal{R}_{T}^{V}\left(p, S_{M}\right)$ contains an open neighborhood of $p$.

d) Symmetrically locally accessible if it is symmetrically locally accessible at every $p \in M$.

e) Controllable if for every $p \in M, \mathcal{R}_{\infty}^{M}\left(p, S_{M}\right)=M$.

The following theorem allows us to check accessibility properties of control systems by simply checking the rank of certain distributions.

Theorem 6.3 (Rank Conditions [18]) : Consider a control system $S_{M}=\left(U_{M}, F_{M}\right)$ on an $n$-dimensional manifold $M$, and let $\mathcal{B}_{M}$ be the associated control bundle. Let $\mathcal{C}_{M}=\operatorname{Lie}\left(\mathcal{B}_{M}\right)$ be the accessibility Lie algebra generated by $\mathcal{B}_{M}$. Then

a) if $\operatorname{dim}\left(\mathcal{C}_{M}(p)\right)=n$, then $S_{M}$ is locally accessible at $p \in$ $M$;

b) if $\operatorname{dim}\left(\mathcal{C}_{M}(p)\right)=n$ for all $p \in M$, then $S_{M}$ is locally accessible;

c) if $\operatorname{dim}\left(\mathcal{C}_{M}(p)\right)=n$ and $\mathcal{B}_{M}(p)$ is symmetric at $p$, that is if $X_{p} \in \mathcal{B}_{M}(p)$ then $-X_{p} \in \mathcal{B}_{M}(p)$, then $S_{M}$ is symmetrically locally accessible at $p \in M$;

d) if $\operatorname{dim}\left(\mathcal{C}_{M}(p)\right)=n$ and $\mathcal{B}_{M}(p)$ is symmetric for all $p \in$ $M$, then $S_{M}$ is symmetrically locally accessible;

e) if $\operatorname{dim}\left(\mathcal{C}_{M}(p)\right)=n, \mathcal{B}_{M}(p)$ is symmetric for all $p \in M$, and $M$ is a connected manifold, then $S_{M}$ is controllable.

We now focus on our problem of interest, namely the propagation of accessibility properties from the original to the abstracted system, and vice versa. One way is immediately given to us by Theorem 4.5 which propagates trajectories from the original to the abstracted system.

Theorem 6.4 (Accessibility Propagation): Let a control system $S_{N}=\left(B_{N}, F_{N}\right)$ be $\Phi$-related to a control system $S_{M}=\left(B_{M}, F_{M}\right)$ with respect to some surjective submersion $\Phi: M \rightarrow N$. Then, for all $p \in M$

$$
\begin{aligned}
\Phi\left(\mathcal{R}_{S_{M}}^{V}(p, T)\right) \subseteq \mathcal{R}_{S_{N}}^{\Phi(V)}(\Phi(p), T) \\
\Phi\left(\mathcal{R}_{T}^{V}\left(p, S_{M}\right)\right) \subseteq \mathcal{R}_{T}^{\Phi(V)}\left(\Phi(p), S_{N}\right)
\end{aligned}
$$

Therefore

a) if $S_{M}$ is locally accessible at $p \in M$, then $S_{N}$ is locally accessible at $\Phi(p) \in N$;

b) if $S_{M}$ is locally accessible, then $S_{N}$ is locally accessible;

c) if $S_{M}$ is symmetric locally accessible at $p \in M$, then $S_{N}$ is symmetric locally accessible at $\Phi(p) \in N$;

d) if $S_{M}$ is symmetric locally accessible, then $S_{N}$ is symmetric locally accessible;

e) if $S_{M}$ is controllable, then $S_{N}$ is controllable.

Proof: Consider any $p \in M$ and let $p^{\prime} \in \mathcal{R}_{S_{M}}^{V}(p, T)$. By assumption there exists trajectory $c_{M}^{U}:[0, T] \rightarrow U_{M}$ of $S_{M}$ with $c_{M}=\pi_{M}\left(c_{M}^{U}\right), c_{M}(0)=p, c_{M}(T)=p^{\prime}$ and for all $0<t \leq T$ we have $c_{M}(t) \in V$. Since $S_{N}$ is $\Phi$-related to $S_{M}$, by Theorem 4.5 there exists trajectory $c_{N}^{U}:[0, T] \rightarrow U_{N}$ of $S_{N}$ with $c_{N}=\pi_{N}\left(c_{N}^{U}\right)$ and $c_{N}(t)=\Phi\left(c_{M}(t)\right)$. Therefore, $c_{N}(0)=\Phi(p), c_{N}(T)=\Phi\left(p^{\prime}\right)$, and $c_{N}(t)=\Phi\left(c_{M}(t)\right) \in$ $\Phi(V)$ for all $0<t \leq T$. Thus, $\left.\Phi\left(p^{\prime}\right) \in \mathcal{R}_{S_{N}}^{\Phi(V)}(\Phi(p), T)\right)$ which proves (11). Having established (11), then (12) as well as a), b), c), d), and e) follow immediately using straightforward topological arguments.

Note that Theorem 6.4 is true for any map $\Phi$ as long as it is a smooth surjective submersion. Furthermore, Theorem 6.4 holds for any two $\Phi$-related systems, not only for the canonical construction of Definition 5.4. A different but equivalent proof of Theorem 6.4 would propagate the accessibility Lie algebra of $S_{M}$ through the epimorphism $T \Phi$.

Whereas Theorem 6.4 propagates accessibility from the original to the abstracted system, from a hierarchical perspective, the reverse question is the complexity reducing direction. In other words, checking accessibility of the abstracted system should be equivalent to checking accessibility of the original, more complicated, system. We shall call such property preserving abstractions consistent abstractions.

This question will be answered for the canonical construction of Definition 5.4. We begin with the following proposition.

Proposition 6.5: Consider an affine control system $S_{M}=$ $\left(U_{M}, F_{M}\right)$ and its associated affine control bundle $\mathcal{A}_{M}$ on a manifold $M$. Let $\Phi: M \rightarrow N$ be a surjective submersion where $N$ is an embedded submanifold of $M$. Use Definition 5.4 to construct control system $\widetilde{S}_{M}$ on $M$ with control bundle $\tilde{\mathcal{A}}_{M}$, and $S_{N}=\left(U_{N}, F_{N}\right)$ on $N$ that is canonically $\Phi$-related to $S_{M}$. Furthermore, assume that

$$
\operatorname{Ker}(T \Phi) \subseteq \operatorname{Lie}\left(\mathcal{A}_{M}\right)
$$

Then, the following hold.

a) $\operatorname{Lie}\left(\tilde{\mathcal{A}}_{M}\right)=\operatorname{Lie}\left(\mathcal{A}_{M}\right)$.

b) For every $p \in M$, open set $V \subset M$, and $T>0$

$$
\mathcal{R}_{T}^{V}\left(p, S_{M}\right)=\mathcal{R}_{T}^{V}\left(p, \tilde{S}_{M}\right)
$$

c) For every $q \in N$ we have

$$
\mathcal{A}_{N}(q)=\tilde{\mathcal{A}}_{M}(q) \cap T_{q} N
$$

d) For every $q \in N$, open set $W \subset N$, and $T>0$

$$
\mathcal{R}_{T}^{W}\left(q, S_{N}\right)=\mathcal{R}_{T}^{\Phi^{-1}(W)}\left(q, \widetilde{S}_{M}\right) \cap N
$$


e) For every $q \in N$, open set $W \subset N$, and $T>0$

$$
\mathcal{R}_{T}^{W}\left(q, S_{N}\right)=\mathcal{R}_{T}^{\Phi^{-1}(W)}\left(q, S_{M}\right) \cap N
$$

\section{Proof:}

a) Since $K \subseteq \operatorname{Lie}\left(\mathcal{A}_{M}\right)$, we have $\tilde{\mathcal{A}}_{M} \subseteq \operatorname{Lie}\left(\mathcal{A}_{M}\right)$, which implies that $\operatorname{Lie}\left(\tilde{\mathcal{A}}_{M}\right) \subseteq \operatorname{Lie}\left(\mathcal{A}_{M}\right)$. The opposite inclusion follows from $\mathcal{A}_{M} \subseteq \tilde{\mathcal{A}}_{M}$.

b) Follows from a).

c) Recall that $K \subset \tilde{\Delta}_{M}$ and $T_{q} \Phi$ is the projection $T_{q} M \rightarrow$ $T_{q} N$ in the direction of $K$. Then, c) follows without difficulty from these facts.

d) Let $q \in N$ be arbitrary and suppose $q^{\prime} \in \mathcal{R}_{T}^{W}\left(q, S_{N}\right)$. Then there exists an $S_{N}$-trajectory $c$ from $q$ to $q^{\prime}$ with $c(t) \in W$ for $0<t \leq T$. By c), $c$ is also an $\tilde{S}_{M}$-trajectory and it clearly lies in $W \subset N$. Thus, $q^{\prime} \in \mathcal{R}_{T}^{W}\left(q, \widetilde{S}_{M}\right) \subset$ $\mathcal{R}_{T}^{\Phi^{-1}(W)}\left(q, \tilde{S}_{M}\right)$ which proves one direction.

Now suppose that $q^{\prime} \in \mathcal{R}_{T}^{\Phi^{-1}(W)}\left(q, \widetilde{S}_{M}\right) \cap N$. Then there exists an $\tilde{S}_{M}$-trajectory $c$ (not necessarily in $N$ ) from $q$ to $q^{\prime}$ with $c(t) \in \Phi^{-1}(W)$ for all $0<t \leq T$. But then $\Phi(c)$ is an $S_{N^{-}}$-trajectory where $q, q^{\prime} \in N, \Phi(c)$ connects $p$ and $q$, and $\Phi(c)(t) \in W$ for all $0<t \leq T$. Therefore, $q^{\prime} \in \mathcal{R}_{T}^{W}\left(q, S_{N}\right)$ which completes the proof.

e) Follows from b) and d).

The following theorem is an immediate consequence of the preceding result.

Theorem 6.6 (Accessibility Equivalence): Consider an affine control system $S_{M}=\left(U_{M}, F_{M}\right)$ and its associated affine control bundle $\mathcal{A}_{M}$ on a manifold $M$, and let $\Phi: M \rightarrow N$ be a surjective submersion. Use Definition 5.4 to construct a control system $S_{N}=\left(U_{N}, F_{N}\right)$ on $N$ that is canonically $\Phi$-related to $S_{M}$. Furthermore, assume that

$$
\operatorname{Ker}(T \Phi) \subseteq \operatorname{Lie}\left(\mathcal{A}_{M}\right)
$$

Then

a) $S_{N}$ is locally accessible at $q \in N$ if and only if $S_{M}$ is locally accessible at every $p \in \Phi^{-1}(q)$;

b) $S_{N}$ is locally accessible if and only if $S_{M}$ is;

c) $S_{N}$ is symmetric locally accessible at $q \in N$ if and only if $S_{M}$ is symmetric locally accessible at every $p \in \Phi^{-1}(q)$;

d) $S_{N}$ is symmetrically locally accessible if and only if $S_{M}$ is;

e) $S_{N}$ is controllable if and only if $S_{M}$ is.

Therefore, if $S_{N}$ is $\Phi$-related to $S_{M}$ using the canonical construction described in Definition 5.4, and condition (13) is satisfied, then $S_{N}$ is a consistent abstraction of $S_{M}$.

Condition (13) can be used in guiding the selection of the abstraction mapping $\Phi(x)$. Note that (13) can always be satisfied as long as inputs exist. For example, for the affine control system

$$
\dot{x}=F(x)+G(x) u
$$

we can always choose a map $\Phi(x)$ whose derivative satisfies the condition $\operatorname{Ker}(T \Phi)=G(x)$, as long as $G(x)$ does not vanish. In this case, we are only ignoring directions that are directly controlled, therefore controllable, and condition (13) is automatically satisfied. The fact that the presence of control makes consistent abstraction possible clearly demonstrates the complexity reducing properties of control systems.

\section{COROLLARIES}

In this section, we illustrate the construction of Definition 5.4 and apply Theorem 6.6 for various classes of affine control systems. We begin by recovering the results for linear systems that were obtained in [20].

\section{A. Linear Systems}

Consider the linear system

$$
\dot{x}=A x+\sum_{i=1}^{m} b_{i} u_{i} \in A x+\operatorname{span}\left\{b_{1}, \ldots, b_{m}\right\}
$$

where $x \in \mathbb{R}^{n}$, and $b_{1}, \ldots, b_{m} \in \mathbb{R}^{n}$ are constant input vector fields. Suppose our abstraction maps are surjective linear maps $y=\Phi(x)=C x$. Then $C$ has full-row rank, the tangent map $T \Phi$ is simply $C$, and $K=\operatorname{Ker}(T \Phi)=\operatorname{Ker}(C)$. Consider

$$
\begin{aligned}
K & =\operatorname{span}\left\{v_{1}, \ldots, v_{k}\right\}, \\
\mathcal{A}_{M}(x) & =A x+\operatorname{span}\left\{b_{1}, \ldots, b_{m}\right\} .
\end{aligned}
$$

The construction of Definition 5.4 results in

$$
\begin{aligned}
L_{M}= & \operatorname{span}\left\{b_{1}, \ldots, b_{m}\right\} \\
& \cup \operatorname{span}\left\{A v_{1}, \ldots, A v_{k}\right\} \\
& \cup \operatorname{span}\left\{v_{1}, \ldots, v_{k}\right\} \\
\tilde{\mathcal{A}}_{M}(x)= & A x+\operatorname{span}\left\{b_{1}, \ldots, b_{m}\right\} \\
& +\operatorname{span}\left\{v_{1}, \ldots, v_{k}\right\} \\
& +\operatorname{span}\left\{A v_{1}, \ldots, A v_{k}\right\} .
\end{aligned}
$$

Higher order Lie brackets in (7) are clearly zero. The affine distribution $\mathcal{A}_{N}(y)$ at $y=C x$ is

$$
\begin{aligned}
\mathcal{A}_{N}(y)=C A x+\operatorname{span}\left\{C b_{1}, \ldots,\right. & \left.C b_{m}\right\} \\
& +\operatorname{span}\left\{C A v_{1}, \ldots, C A v_{k}\right\} .
\end{aligned}
$$

for any $x \in C^{-1}(y)$. Since $C$ has full row rank, we can choose $x=C^{+} y$ where $C^{+}=C^{T}\left(C C^{T}\right)^{-1}$ is the Moore-Penrose pseudoinverse of $C$. Therefore, the canonically $C$-related system for any linear surjective map $y=C x$ is

$$
\begin{aligned}
\mathcal{A}_{N}(y)=C A C^{+} y+\operatorname{span}\left\{C b_{1}, \ldots,\right. & \left.C b_{m}\right\} \\
& +\operatorname{span}\left\{C A v_{1}, \ldots, C A v_{k}\right\}
\end{aligned}
$$

or more compactly

$$
\dot{y}=F y+G v
$$

where

$$
\begin{aligned}
& F=C A C^{+} \\
& G=\left[\begin{array}{lllllll}
C b_{1} & \ldots & C b_{m} & C A v_{1} & \ldots & C A v_{k}
\end{array}\right] .
\end{aligned}
$$


In order to propagate accessibility properties, the linear abstraction map must satisfy the consistency condition (13) which in the linear context becomes

$$
\begin{aligned}
\operatorname{Ker}(C) & \subseteq \operatorname{Lie}\left(A x, b_{1}, \ldots, b_{m}\right) \\
& =\operatorname{span}\left[\begin{array}{llll}
B & A B & \ldots & \left.A^{n-1} B\right]+\operatorname{span}\{A x\}
\end{array}\right.
\end{aligned}
$$

Condition (16) can always be satisfied as long as $B \neq 0$ since we can always choose $\operatorname{Ker}(C)=\operatorname{span}[B]$. In order words, we can always obtain accessibility preserving abstractions as long as there are control inputs. Under these conditions, Theorem 6.6 directly implies that local accessibility of (15) is equivalent to local accessibility of (14). In fact, from Theorem 2.5, condition (16) propagates not only local accessibility, but also global controllability [20].

\section{B. Bilinear Systems}

Consider the bilinear system

$$
\dot{x}=A x+\sum_{i=1}^{m}\left(B_{i} \cdot x\right) u_{i}
$$

where $x \in \mathbb{R}^{n}$, and $A, B_{1}, \ldots, B_{m} \in \mathbb{R}^{n \times n}$. Note that the reachable set from the origin is only the origin. Suppose our aggregation map is again linear $y=\Phi(x)=C x$ and surjective. Then

$$
\begin{aligned}
K & =\operatorname{span}\left\{v_{1}, \ldots, v_{k}\right\}, \\
\mathcal{A}_{M} & =A x+\operatorname{span}\left\{B_{1} x, \ldots, B_{m} x\right\} .
\end{aligned}
$$

The canonical construction results in

$$
\begin{aligned}
L_{M}(x)= & \operatorname{span}\left\{B_{1} x, \ldots, B_{m} x\right\} \\
& +\operatorname{span}\left\{v_{1}, \ldots, v_{k}\right\} \\
& +\operatorname{span}\left\{A v_{1}, \ldots, A v_{k}\right\} \\
\tilde{\mathcal{A}}_{M}(x)= & A x+\operatorname{span}\left\{B_{1} x, \ldots, B_{m} x\right\} \\
& +\operatorname{span}\left\{v_{1}, \ldots, v_{k}\right\} \\
& +\operatorname{span}\left\{A v_{1}, \ldots, A v_{k}\right\} \\
& +\operatorname{span}\left\{B_{1} v_{1}, \ldots, B_{1} v_{k}\right\} \\
& \vdots \\
& +\operatorname{span}\left\{B_{m} v_{1}, \ldots, B_{m} v_{k}\right\} .
\end{aligned}
$$

Second-order Lie brackets between $L_{M}$ and $K$ are zero. Since $v_{1}, \ldots, v_{k} \in \operatorname{Ker}(C)$, and choosing $x=C^{+} y$ results in an affine bundle $\mathcal{A}_{N}$ defined by

$$
\begin{aligned}
\mathcal{A}_{N}(y)= & C A C^{+} y+\operatorname{span}\left\{C B_{1} C^{+} y, \ldots, C B_{m} C^{+} y\right\} \\
& +\operatorname{span}\left\{C A v_{1}, \ldots, C A v_{k}\right\} \\
& +\operatorname{span}\left\{C B_{1} v_{1}, \ldots, C B_{1} v_{k}\right\} \\
& \vdots \\
& +\operatorname{span}\left\{C B_{m} v_{1}, \ldots, C B_{m} v_{k}\right\} .
\end{aligned}
$$

Therefore. the canonically $C$-related system is

$$
\begin{aligned}
\dot{y}=C A C^{+} y+\sum_{i=1}^{m}( & \left.C B_{i} C^{+} y\right) v_{i} \\
& +\sum_{i=1}^{k}\left(C A v_{i}\right) w_{k}+\sum_{i=1}^{m} \sum_{j=1}^{k}\left(C B_{i} v_{j}\right) z_{i j} .
\end{aligned}
$$

In order to propagate accessibility properties, the linear abstraction map must satisfy the consistency condition (13)

$$
\operatorname{Ker}(C) \subseteq \operatorname{Lie}\left(A x, B_{1} x, \ldots, B_{m} x\right)
$$

The Lie algebra $\operatorname{Lie}\left(A x, B_{1} x, \ldots, B_{m} x\right)$ of bilinear systems is spanned by $\left[A, B_{i}\right] x,\left[B_{i}, B_{j}\right] x$ and higher order matrix brackets. Unfortunately, at $x=0$ we have $\operatorname{Lie}\left(A x, B_{1} x, \ldots, B_{m} x\right)=0$, and therefore, a consistent abstraction is obtained only on $\mathbb{R}^{n} \backslash\{0\}$. This is not necessarily the case, however, if one considers bilinear systems of the form

$$
\dot{x}=A x+\sum_{i=1}^{m}\left(B_{i} \cdot x\right) u_{i}+\sum_{i=1}^{k} d_{i} w_{i}
$$

in which case one can consistently abstract some dynamics on $\mathbb{R}^{n}$ by choosing $\operatorname{Ker}(C) \subseteq \operatorname{span}\left\{d_{1}, \ldots, d_{k}\right\}$.

\section{Drift Free Systems}

As a special case of affine control systems, consider the so-called drift free systems

$$
\dot{x}=\sum_{i=1}^{m} g_{i}(x) u_{i} \in \operatorname{span}\left\{g_{1}(x), \ldots, g_{m}(x)\right\}
$$

where $g_{1}(x), \ldots, g_{m}(x)$ are smooth vector fields on $\mathbb{R}^{n}$. In this case, the canonical construction of Definition 5.4 is simplified as the drift term $X_{M}=0$. Therefore, rather than dealing with affine bundles, we now work with standard distributions. This results in the following construction.

Definition 7.1 (Canonical Construction on $N$ ): Let $S_{M}=$ $\left(U_{M}, F_{M}\right)$ be a drift-free control system on a manifold $M$ with distribution $\Delta_{M}$. Let $\Phi: M \rightarrow N$ be a surjective submersion, where $N$ is an embedded submanifold of $M$. Denote by $K$ the vector subbundle of $T M$ defined as

$$
K=\operatorname{Ker}(T \Phi)=\{v \in T M: T \Phi(v)=0\}
$$

Define the distribution $\tilde{\Delta}_{M}$ which is generated by

$$
\Delta_{M} \cup K \cup\left[K, \Delta_{M}\right] \cup\left[K,\left[K, \Delta_{M}\right]\right] \cup \cdots .
$$

The distribution $\Delta_{N}$ on $N$ defined by

$$
\Delta_{N}(q)=T_{p} \Phi\left(\tilde{\Delta}_{M}(p)\right)
$$

for any $p \in \Phi^{-1}(q)$, is canonically $\Phi$-related to $\Delta_{M}$. Any control system $S_{N}=\left(U_{N}, F_{N}\right)$ with distribution $\Delta_{N}$ is said to be canonically $\Phi$-related to $S_{M}$.

The canonical construction of Definition 5.4 ensures that the abstraction of an affine control system is affine. Similarly, the 
canonical construction of Definition 7.1 ensures that the abstraction of drift free control systems is also drift free. As an example, consider the unicycle model

$$
\left[\begin{array}{c}
\dot{x} \\
\dot{y} \\
\dot{\theta}
\end{array}\right]=\left[\begin{array}{c}
\cos (\theta) \\
\sin (\theta) \\
0
\end{array}\right] v+\left[\begin{array}{l}
0 \\
0 \\
1
\end{array}\right] \omega
$$

and consider the abstracting map which simply ignores $\theta$, that is $\Phi(x, y, \theta)=(x, y)$. The construction of Definition 7.1 results in

$$
\left[\begin{array}{c}
\dot{x} \\
\dot{y}
\end{array}\right]=\left[\begin{array}{c}
\cos (\theta) \\
\sin (\theta)
\end{array}\right] v_{1}+\left[\begin{array}{c}
\sin (\theta) \\
-\cos (\theta)
\end{array}\right] v_{2}
$$

for any choice of $\theta$. Choosing $\theta=0$ results in

$$
\begin{aligned}
& \dot{x}=v_{1} \\
& \dot{y}=v_{2} .
\end{aligned}
$$

Note that the canonical construction preserves the drift free structure of the system. Furthermore, since

$$
\operatorname{Ker}(T \Phi)=\operatorname{span}\left\{\left[\begin{array}{l}
0 \\
0 \\
1
\end{array}\right]\right\} \subseteq \operatorname{span}\left\{\left[\begin{array}{c}
\cos (\theta) \\
\sin (\theta) \\
0
\end{array}\right],\left[\begin{array}{l}
0 \\
0 \\
1
\end{array}\right]\right\}
$$

system (22) is a consistent abstraction of the unicycle model (21). Therefore the unicycle model (21) is locally accessible if and only if system (22) is locally accessible, which is trivially true. The above abstraction of the nonholomic unicycle by a two dimensional integrator is exactly in the spirit of [25], where topological properties for collision avoidance of the models are also considered in detail.

\section{Strict Feedback Systems}

Consider the class of strict feedback systems used in backstepping designs [12], which have the following block triangular structure

$$
\begin{aligned}
\dot{x}_{1} & =f_{1}\left(x_{1}\right)+g_{1}\left(x_{1}\right) x_{2} \\
\dot{x}_{2} & =f_{2}\left(x_{1}, x_{2}\right)+g_{2}\left(x_{1}, x_{2}\right) x_{3} \\
& \vdots \\
\dot{x}_{n} & =f_{n}\left(x_{1}, x_{2}, \ldots, x_{n}\right)+g_{n}\left(x_{1}, x_{2}, \ldots, x_{n}\right) u
\end{aligned}
$$

where $x_{i} \in \mathbb{R}^{n_{i}}$ and all maps $f_{i}, g_{i}$ are smooth. For notational simplicity, we present the canonical construction for $n=2$, that is

$$
\left[\begin{array}{c}
\dot{x}_{1} \\
\dot{x}_{2}
\end{array}\right]=\left[\begin{array}{c}
f_{1}\left(x_{1}\right)+g_{1}\left(x_{1}\right) x_{2} \\
f_{2}\left(x_{1}, x_{2}\right)
\end{array}\right]+\left[\begin{array}{c}
0 \\
g_{2}\left(x_{1}, x_{2}\right)
\end{array}\right] u
$$

and therefore, the affine bundle is

$$
\begin{aligned}
\mathcal{A}_{M}\left(x_{1}, x_{2}\right)= & {\left[\begin{array}{c}
f_{1}\left(x_{1}\right)+g_{1}\left(x_{1}\right) x_{2} \\
f_{2}\left(x_{1}, x_{2}\right)
\end{array}\right] } \\
& +\operatorname{span}\left\{\left[\begin{array}{c}
0 \\
g_{2}\left(x_{1}, x_{2}\right)
\end{array}\right]\right\} .
\end{aligned}
$$

Suppose our abstraction map is the a simple projection $y=$ $\Phi\left(x_{1}, x_{2}\right)=x_{1}$. Then

$$
\begin{gathered}
y=\left[\begin{array}{ll}
I & 0
\end{array}\right]\left[\begin{array}{l}
x_{1} \\
x_{2}
\end{array}\right]=x_{1} \\
K=\operatorname{Ker}(T \Phi)=\operatorname{span}\left\{\left[\begin{array}{l}
0 \\
I
\end{array}\right]\right\} .
\end{gathered}
$$

The canonical construction results in $L_{M}=\Delta_{M} \cup K \cup$ $\left[K, X_{M}\right]$, where

$$
\begin{aligned}
\Delta_{M} & =\operatorname{span}\left\{\left[\begin{array}{c}
0 \\
g_{2}\left(x_{1}, x_{2}\right)
\end{array}\right]\right\} \\
{\left[K, X_{M}\right] } & =\left[\left[\begin{array}{l}
0 \\
I
\end{array}\right],\left[\begin{array}{c}
f_{1}+g_{1} \cdot x_{2} \\
f_{2}
\end{array}\right]\right]=\left[\begin{array}{c}
g_{1} \\
\frac{\partial f_{2}}{\partial x_{2}}
\end{array}\right]
\end{aligned}
$$

and therefore, $\left[K, L_{M}\right]$ consists of

$$
\begin{aligned}
{\left[K, \Delta_{M}\right] } & =\operatorname{span}\left\{\left[\begin{array}{c}
0 \\
\frac{\partial g_{2}}{\partial x_{2}}
\end{array}\right]\right\} \\
{\left[K,\left[K, X_{M}\right]\right] } & =\operatorname{span}\left\{\left[\begin{array}{c}
0 \\
\frac{\partial^{2} f_{2}}{\partial x_{2}^{2}}
\end{array}\right]\right\} \\
{[K, K] } & =0 .
\end{aligned}
$$

Clearly, $\left[K, L_{M}\right] \subseteq K$. Higher order Lie brackets, even though nonzero, also belong to $K=\operatorname{Ker}(T \Phi)$. Therefore, the construction results in

$$
\begin{aligned}
\tilde{\mathcal{A}}_{M}= & {\left[\begin{array}{c}
f_{1}\left(x_{1}\right)+g_{1}\left(x_{1}\right) x_{2} \\
f_{2}\left(x_{1}, x_{2}\right)
\end{array}\right]+\operatorname{span}\left\{\left[\begin{array}{c}
0 \\
g_{2}\left(x_{1}, x_{2}\right)
\end{array}\right]\right\} } \\
& +\operatorname{span}\left\{\left[\begin{array}{c}
g_{1} \\
\frac{\partial f_{2}}{\partial x_{2}}
\end{array}\right]\right\}+\operatorname{span}\left\{\left[\begin{array}{l}
0 \\
I
\end{array}\right]\right\} \\
& +\operatorname{span}\left\{\left[\begin{array}{c}
0 \\
\frac{\partial g_{2}}{\partial x_{2}}
\end{array}\right]\right\}+\operatorname{span}\left\{\left[\begin{array}{c}
0 \\
\frac{\partial^{2} f_{2}}{\partial x_{2}^{2}}
\end{array}\right]\right\} .
\end{aligned}
$$

Pushing forward $\tilde{\mathcal{A}}_{M}\left(x_{1}, x_{2}\right)$ through $T \Phi$ results in

$$
\mathcal{A}_{N}\left(x_{1}\right)=f_{1}\left(x_{1}\right)+g_{1}\left(x_{1}\right) x_{2}+\operatorname{span}\left\{g_{1}\left(x_{1}\right)\right\}
$$

for any $\left(x_{1}, x_{2}\right) \in \Phi^{-1}\left(x_{1}\right)$. Choosing $\left(x_{1}, 0\right) \in \Phi^{-1}\left(x_{1}\right)$ results in the following abstracted system:

$$
\begin{aligned}
\dot{x}_{1} & =f_{1}\left(x_{1}\right)+\operatorname{span}\left\{g_{1}\left(x_{1}\right)\right\} \\
& =f_{1}\left(x_{1}\right)+g_{1}\left(x_{1}\right) x_{2}
\end{aligned}
$$

where $x_{2}$ is now thought of as a virtual input. The above calculation also shows that for strict feedback systems, if $x_{2}$ is to be abstracted, then one can simply eliminate the differential equation associated with $x_{2}$. Therefore, the triangular nature of strict feedback systems make the computations for the canonical construction very simple.

In order to propagate accessibility, the consistency condition (13) must be satisfied. This means that

$$
\begin{aligned}
\operatorname{Ker}(T \Phi) \subseteq \operatorname{Lie}\left(\mathcal{A}_{M}\right) \\
\Rightarrow \operatorname{span}\left\{\left[\begin{array}{l}
0 \\
I
\end{array}\right]\right\} \subseteq \operatorname{Lie}\left(\left[\begin{array}{c}
f_{1}+g_{1} x_{2} \\
f_{2}
\end{array}\right],\left[\begin{array}{c}
0 \\
g_{2}
\end{array}\right]\right) .
\end{aligned}
$$


From (28), it is clear that if $g_{2}\left(x_{1}, x_{2}\right) \neq 0$ for all $x_{1}, x_{2}$, then the consistency condition is trivially satisfied and the the local accessibility of (24) is equivalent to the local accessibility of (27). If $g_{2}\left(x_{1}, x_{2}\right)=0$ for some $x_{1}, x_{2}$, then the consistency condition may be satisfied by $f_{2}\left(x_{1}, x_{2}\right)$ or by using higher order Lie brackets. For example, the first-order Lie bracket contains

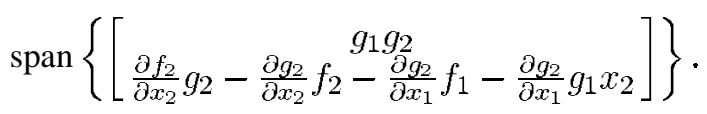

Therefore, the consistency condition is automatically satisfied if

$\operatorname{span}\left\{g_{2}, f_{2}, \frac{\partial f_{2}}{\partial x_{2}} g_{2}-\frac{\partial g_{2}}{\partial x_{2}} f_{2}-\frac{\partial g_{2}}{\partial x_{1}} f_{1}-\frac{\partial g_{2}}{\partial x_{1}} g_{1} x_{2}\right\}=\mathbb{R}^{n_{2}}$

for all $x_{1}, x_{2} \in \mathbb{R}^{n_{1}+n_{2}}$. If this is not satisfied, then higher order Lie brackets may be used.

Some classes of strict feedback systems deserve special attention.

\section{E. Nonlinear Systems With Appended Linear Dynamics}

Consider the following class of systems:

$$
\begin{aligned}
& \dot{x}_{1}=f\left(x_{1}\right)+g\left(x_{1}\right) x_{2} \\
& \dot{x}_{2}=A x_{2}+B u
\end{aligned}
$$

where $x_{1} \in \mathbb{R}^{n_{1}}, x_{2} \in \mathbb{R}^{n_{2}}, u \in \mathbb{R}^{n_{u}}, f, g$ are smooth maps, and $A, B$ are matrices of appropriate size. Such systems frequently arise in mechanical systems with nonlinear kinematics but linear actuator dynamics. In studying the local accessibility of such systems, rather than computing the full-blown accessibility Lie algebra, one would like to decompose the analysis in order to reduce the complexity.

System (29) can be thought of as a strict feedback system with considerably more structure since $f_{2}\left(x_{1}, x_{2}\right)=A x_{2}$ and $g_{2}\left(x_{1}, x_{2}\right)=B$. Consider again the simple projection map $\Phi\left(x_{1}, x_{2}\right)=x_{1}$ which ignores the linear dynamics. The canonical construction of Theorem 5.5 proceeds in the same way as for strict feedback systems and results in the $\Phi$-related system

$$
\dot{x}_{1}=f\left(x_{1}\right)+g\left(x_{1}\right) x_{2}
$$

where $x_{2} \in \mathbb{R}^{n_{2}}$ is now an input.

Local accessibility of (30) is equivalent to the local accessibility of (29) if the consistency condition (13) is satisfied. The special structure of system (29), and some algebra reveals the following consistency condition:

$$
\begin{aligned}
\operatorname{Ker}(T \Phi) & =\operatorname{span}\left\{\left[\begin{array}{l}
0 \\
I
\end{array}\right]\right\} \\
& \subseteq \operatorname{span}\left\{\left[\begin{array}{cccc}
\text { irrelevant terms } \\
B & A B & \ldots & A^{n_{2}} B
\end{array}\right]\right\} .
\end{aligned}
$$

In other words, if the pair $(A, B)$ is controllable, then we can simply ignore the linear part of the system, and local accessibility of (30) is equivalent to the local accessibility of (29).
Therefore, the accessibility properties of system (29) truly decompose to the controllability property of the linear subsystem, and the accessibility property of the nonlinear subsystem.

\section{F. Linear Systems With Appended Nonlinearities}

Conversely, consider the following class of systems:

$$
\begin{aligned}
& \dot{x}_{1}=A x_{1}+B x_{2} \\
& \dot{x}_{2}=f_{2}\left(x_{2}\right)+g_{2}\left(x_{2}\right) u
\end{aligned}
$$

where $x_{1} \in \mathbb{R}^{n_{1}}, x_{2} \in \mathbb{R}^{n_{2}}, u \in \mathbb{R}^{n_{u}}, f, g$ are smooth maps, and $A, B$ are matrices of appropriate dimension. In this case, the abstracting map $\Phi\left(x_{1}, x_{2}\right)=x_{1}$ ignores the nonlinear part of the system. System (31) can be thought of as system in strict feedback form with special structure. Therefore, the canonical construction results in the abstracted model

$$
\dot{x}_{1}=A x_{1}+B x_{2} \text {. }
$$

Again the structure of (29) and some algebra lead to the following form for the consistency condition:

$$
\begin{aligned}
\operatorname{Ker}(T \Phi) & =\operatorname{span}\left\{\left[\begin{array}{l}
0 \\
I
\end{array}\right]\right\} \\
& \subseteq \operatorname{span}\left\{\left[\begin{array}{c}
\text { irrelevant terms } \\
\operatorname{Lie}\left(f_{2}, g_{2}\right)
\end{array}\right]\right\} .
\end{aligned}
$$

Therefore, if the nonlinear subsystem is locally accessible, that is $\operatorname{Lie}\left(f_{2}, g_{2}\right)=\mathbb{R}^{n_{2}}$, then the local accessibility of the nonlinear system (31) is equivalent to the controllability of the linear system (32).

\section{CONCLUSION}

In this paper, consistent abstractions of affine control systems were considered. In particular, we provided constructive methods for abstracting affine control systems with respect to smooth surjective maps. Our construction is structure preserving in the sense that affine control systems are abstracted by affine control systems. Furthermore, we characterized abstraction maps that result in preserving the property of local accessibility from the abstracted model to the original model. Our framework was then applied to various classes of nonlinear control systems including linear, bilinear, drift free, and strict feedback systems.

We believe that there is a clear research agenda which focuses on classes of systems as well as properties of interest and characterizes the abstracting maps that preserve the properties of interest for the particular class under consideration. For example, obtaining consistent abstractions for nonlinear systems with respect to stabilizability would be helpful in better understanding backsteppable systems. For hierarchical controller design, refining the controller design from the abstracted level to the more complicated model is a challenge. For linear systems, this was recently achieved in [19] from which we can extract as a special case the the hierarchical stabilization algorithm of [24]. Other properties of interest include trajectory optimality, preserving Hamiltonian structure [27], and the propagation of state and input constraints. 


\section{ACKNOWLEDGMENT}

The authors would like to thank P. Tabuada and G. Lafferriere for many inspiring discussions on the problem of system abstraction.

\section{REFERENCES}

[1] R. Abraham, J. Marsden, and T. Ratiu, Manifolds, Tensor Analysis and Applications, Applied Mathematical Sciences. New York: SpringerVerlag, 1988.

[2] R. Alur, T. Henzinger, G. Lafferriere, and G. J. Pappas, "Discrete abstractions of hybrid systems," Proc. IEEE, vol. 88, pp. 971-984, July 2000.

[3] A. Antoulas, "Approximation of linear dynamical systems," in Wiley Encyclopedia of Electrical and Electronics Engineering, J. G. Webster, Ed. New York: Wiley, 1999, vol. 11, pp. 403-422.

[4] P. J. Antsaklis, J. A. Stiver, and M. Lemmon, "Hybrid system modeling and autonomous control systems," in Hybrid Systems, R. L. Grossman, A. Nerode, A. P. Ravn, and H. Rischel, Eds. New York: Springer-Verlag, 1993, vol. 736, Lecture Notes in Computer Science, pp. 366-392.

[5] M. Aoki, "Control of large scale dynamic systems by aggregation," IEEE Trans. Automat. Contr., vol. AC-13, pp. 246-253, June 1968.

[6] R. Brockett, "Control theory and analytical mechanics," in Geometric Control Theory, Lie Groups: History, Frontiers and Applications, C. Martin and R. Hermann, Eds. Brookline, MA: Mathematical Scientific Press, 1977, pp. 1-46.

[7] P. Caines and Y. J. Wei, "The hierarchical lattices of a finite state machine," Syst. Control Lett., vol. 25, pp. 257-263, 1995.

[8] _ - "Hierarchical hybrid control systems: A lattice theoretic formulation," IEEE Trans. Automat. Contr., vol. 43, pp. 501-508, Apr. 1998

[9] P. Cousot and R. Cousot, "Systematic design of program analysis framework," in Proc. 6th ACM Symp. Principles Programming Languages, San Antonio, TX, Jan. 1979, pp. 269-282.

[10] J. E. R. Cury, B. H. Krogh, and T. Niinomi, "Synthesis of supervisory controllers for hybrid systems based on approximating automata," IEEE Trans. Automat. Contr., vol. 43, pp. 564-568, Apr. 1998.

[11] P. M. Van Dooren, "The generalized eigenstructure problem in linear system theory," IEEE Trans. Automat. Contr., vol. AC-26, pp. 111-129, Jan. 1981

[12] M. Kristic, I. Kanellakopoulos, and P. Kokotovic, Nonlinear and Adaptive Control Design, Adaptive and Learning Systems for Signal Processing, Communications and Control. New York: Wiley, 1995.

[13] C. P. Kwong, "Optimal chained aggregation for reduced order modeling," Int. J. Control, vol. 35, no. 6, pp. 965-982, 1982.

[14] G. Lafferriere, G. J. Pappas, and S. Sastry, "O-minimal hybrid systems," Math. Control, Signals, Syst., vol. 13, no. 1, pp. 1-21, Mar. 2000.

[15] A. Laub, R. Patel, and P. Van Dooren, Numerical Linear Algebra for Systems and Control. Piscataway, NJ: IEEE Press, 1993.

[16] C. Loiseaux, S. Graf, J. Sifakis, A. Bouajjani, and S. Bensalem, "Property preserving abstractions for the verification of concurrent systems," in Formal Methods in Systems Design. Boston, MA: Kluwer, 1995, vol. 6, pp. 1-35.

[17] R. Milner, Communication and Concurrency. Upper Saddle River, NJ: Prentice-Hall, 1989.

[18] H. Nijmeijer and A. J. van der Schaft, Nonlinear Dynamical Control Systems. New York: Springer-Verlag, 1990.

[19] G. J. Pappas and G. Lafferriere, "Hierarchies of stabilizability preserving linear systems," in Proc. 40th IEEE Conf. Decision Control, Orlando, FL, Dec. 2001, pp. 2081-2086.

[20] G. J. Pappas, G. Lafferriere, and S. Sastry, "Hierarchically consistent control systems," IEEE Trans. Automat. Contr., vol. 45, pp. 1144-1160, June 2000.

[21] G. J. Pappas and S. Sastry, "Toward continuous abstractions of dynamical and control systems," in Hybrid Systems IV, P. Antsaklis, W. Kohn, A. Nerode, and S. Sastry, Eds. Berlin, Germany: Springer-Verlag, 1997, vol. 1273, Lecture Notes in Computer Science, pp. 329-341.
[22] G. J. Pappas and S. Simic, "Consistent hierarchies of nonlinear abstractions," in Proc. 39th IEEE Conf. Decision Control, Sydney, Australia, Dec. 2000 , pp. $4379-4384$

[23] J. Raisch and S. D. O'Young, "Discrete approximations and supervisory control of continuous systems," IEEE Trans. Automat. Contr, vol. 43, pp. 569-573, Apr. 1998

[24] Y. Saad, "Projection and deflation methods for partial pole assignment in linear state feedback," IEEE Trans. Automat. Contr., vol. 33, pp. 290-297, Mar. 1988.

[25] S. Sekhavat and J. P. Laumond, "Topological property for collision-free nonholonomic motion planning: The case of sinusoidal inputs for chained form systems," IEEE Trans. Robot. Automat., vol. 14, pp. 671-680, Oct. 1998

[26] S. Stankovic and D. Siljak, "Contractibility of overlapping decentralized control," Syst. Control Lett., vol. 44, no. 3, pp. 189-200, Oct. 2001.

[27] P. Tabuada and G. J. Pappas, "Abstractions of hamiltonian control systems," in Proc. 39th IEEE Conf. Decision Control, Orlando, FL, Dec. 2001, pp. 3394-3399.

[28] P. van Dooren, "Gramian based model reduction of large scale dynamical systems," in Numerical Analysis 1999. Boca Raton, FL: CRC Press, 2000, pp. 231-247.

[29] K. C. Wong and W. M. Wonham, "Hierarchical control of discrete-event systems," Discrete Event Dyna. Syst., vol. 6, pp. 241-273, 1995.

[30] H. Zhong and W. M. Wonham, "On the consistency of hierarchical supervision in discrete-event systems," IEEE Trans. Automat. Contr., vol. 35, pp. 1125-1134, Oct. 1990.

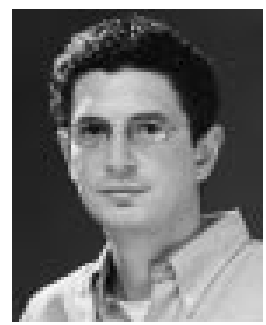

George J. Pappas (S'91-M'98) received the B.S. and M.S. degrees in computer and systems engineering, both from Rensselaer Polytechnic Institute, Troy, NY, and the Ph.D. degree from the Department of Electrical Engineering and Computer Science, the University of California at Berkeley, in 1991, 1992, and 1998 , respectively.

In 1994, he was a Graduate Fellow at the Division of Engineering Science of Harvard University. He was a Postdoctoral Researcher at the University of California at Berkeley and the University of Pennsylvania, Philadelphia. Currently, he is an Assistant Professor and Graduate Group Chair in the Department of Electrical Engineering at the University of Pennsylvania, where he also holds a secondary appointment in the Department of Computer and Information Sciences. His research interests include hierarchical control systems, embedded hybrid systems, distributed control systems, nonlinear control systems, geometric control theory, with applications to flight management systems, robotics, and unmanned aerial vehicles.

Dr. Pappas was the recipient of the 2002 National Science Foundation CAREER award and the 1999 Eliahu Jury Award for Excellence in Systems Research from the Department of Electrical Engineering and Computer Sciences at the University of California at Berkeley. He was also a finalist for the Best Student Paper Award at the 1998 IEEE Conference on Decision and Control.

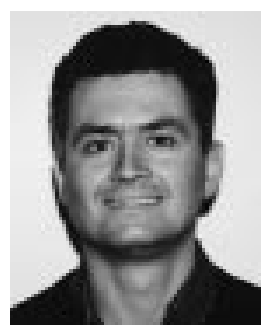

Slobodan Simić received the B.S. degree from the University of Belgrade, Yugoslavia, and the Ph.D. degree from the University of California at Berkeley, both in mathematics, in 1988 and 1995, respectively.

He was a Visiting Assistant Professor at the University of Illinois at Chicago from 1995 to 1996, and an Assistant Professor at the University of Southern California, Los Angeles, from 1996 to 1999, both in the Mathematics Departments. He is currently a Postdoctoral Researcher and Lecturer at the Department of Electrical Engineering and Computer Science, the University of California at Berkeley. His research interests are geometric theory of dynamical, control, and hybrid systems, sensor networks, and quantum computing. 